food. Managing disease-specific symptoms associated with cardiovascular disease, diabetes, mental health, and respiratory diseases were primary concerns following a disaster. Stress and anxiety, loss of sleep, weakness or fatigue and shortness of breath were common concerns for all noncommunicable diseases. Those dependent on care from others were most worried about shortness of breath and slow healing sores. Accessing medication and medical services were priorities for all patients post-disaster.

Discussion: The key influences on successful self-management post disaster for people with noncommunicable diseases must be reflected in disaster plans and strategies. Achieving this will reduce exacerbations or complications of disease and decrease demand for emergency health care post-disaster.

Prehosp. Disaster Med. 2019;34(Suppl. 1):s47-s48

doi:10.1017/S1049023X19001122

\section{Disaster Risk Reduction and Health: The Potential of Health Registers for Public Health Monitoring}

Dr. Michel Dückers ${ }^{1,2}$, Dr. Filip Arnberg ${ }^{3}$, Dr. Christos Baliatsas ${ }^{1}$, Dr. Lennart Reifels ${ }^{4}$, Dr. Lise Stene ${ }^{5}$, Dr. Joris Yzermans ${ }^{1}$

1. Nivel - Netherlands Institute for Health Services Research, Utrecht, Netherlands

2. Impact, National Knowledge and Advice Centre for Psychosocial Care Concerning Critical Incidents, Partner in Arq Psychotrauma Expert Group, Diemen, Netherlands

3. Uppsala University, Uppsala, Sweden

4. Melbourne School of Population and Global Health, University of Melbourne, Victoria, Australia

5. Norwegian Centre for Violence and Traumatic Stress Studies, Oslo, Norway

Introduction: The Sendai Framework seeks to substantially reduce disaster risk and losses in lives, livelihoods, health, and other assets including persons, communities, and countries. The framework focuses on reducing mortality while increasing population wellbeing, early warning, and promotion of health systems resilience. The use of scientific evidence to inform policy and formulate effective initiatives and interventions is crucial to disaster risk reduction within health. Different instruments and methodologies are available to guide policy and operations. The potential value of routinely collected patient data from health registers is that they can provide pre-event health and comparison group data without burdening affected populations. Aim: The current contribution aims to illustrate how health registers can help monitor the health impact of natural and human-made disasters.

Methods: Patient data from health registers of general practitioners and other health professionals, sometimes combined with other registers and data sources, have been utilized to monitor the health impact of disasters and environmental hazards in the Netherlands, Norway, and Sweden since 2000.

Results: Health registers allowed monitoring of mental health problems, medically unexplained symptoms, chronic health problems, and social problems. These were compared to groups not directly exposed. The health impact and care utilization was tracked after the fireworks explosion in Enschede affecting inhabitants of the neighborhood (2000; data range 1999-2005), children and parents after the Volendam café fire (2001; data range 2000-2006), Swedish survivors of the Tsunami in Southeast Asia (2004; data range 2004-2010), and parents of children affected by the terrorist attack on Utøya (2011; data range 2008-2014).

Discussion: Health systems with registers have an important advantage when it comes to the potential for monitoring population health, and perhaps offer early warnings of pandemics. However, data generation should be closely connected to policy-making before and during the planning and evaluation of public health intervention.

Prehosp. Disaster Med. 2019;34(Suppl. 1):s48

doi:10.1017/S1049023X19001134

\section{The Effect of Natural Disasters on Cancer Care:} A Systematic Review

Dr. Ralph Xiu-gee Man ${ }^{1}$, Dr. David Lack2,

Dr. Charlotte Wyatt ${ }^{3}$, Prof. Virginia Murray ${ }^{4}$

1. Princess Alexandra Hospital, Brisbane, Australia

2. Department of Emergency medicine, Hervey Bay Hospital, Hervey Bay, Australia

3. Royal Brisbane and Women's hospital, Brisbane, Australia

4. Global Disaster Risk Reduction, Public Health England, London, UK

Introduction: As the incidence of cancer and the frequency of extreme weather events rise, disaster mitigation is becoming increasingly relevant to oncology care.

Aim: To investigate the effect of natural disasters on cancer care and the associated health effects on patients with cancer through the means of a systematic review.

Methods: Between database inception and November 12, 2016, Embase, ScienceDirect, MEDLINE, Scopus, PsycINFO, Web of Science, and CINAHL were searched for articles. Those identifying the effect of natural disasters on oncology services, or the associated health implications for patients with cancer, were included. Only articles published in English were included. Data extraction was done by two authors independently and then verified by all authors. The effects of disaster events on oncology services, survival outcomes, and psychological issues were assessed.

Results: Natural disasters cause substantial interruption to the provision of oncology care. Of the 4,593 studies identified, only 85 articles met all the eligibility criteria. Damage to infrastructure, communication systems, medication, and medical record losses substantially disrupt oncology care. The effect of extreme weather events on survival outcomes is limited to only a small number of studies, often with inadequate follow-up periods.

Discussion: To the best the authors' knowledge, this is the first systematic review to assess the existing evidence base on the health effects of natural disaster events on cancer care. Disaster planning must begin to take into consideration patients with cancer.

Prehosp. Disaster Med. 2019;34(Suppl. 1):s48

doi:10.1017/S1049023X19001146 